\section{Preprint}

not peer reviewed

\title{
Unlocking sports medicine research data while maintaining participant privacy via synthetic datasets
}

Received: 20 August 2020

For correspondence: john.warmenhoven@hotmail.com

John Warmenhoven ${ }^{1,2}$, Andrew J. Harrison ${ }^{3}$, Daniel S. Quintana ${ }^{4}$, Giles Hooker ${ }^{5,6}$, Edward Gunning ${ }^{7}$, Norma Bargary $^{7}$

${ }^{1}$ Exercise \& Sport Science, University of Sydney, Sydney, Australia, ${ }^{2}$ Australian Institute of Sport, Canberra, Australia, ${ }^{3}$ Biomechanics Research Unit, University of Limerick, Limerick, Ireland, ${ }^{4}$ Norwegian Centre for Mental Disorders Research (NORMENT), Oslo University Hospital \& Institute of Clinical Medicine, University of Oslo, Oslo, Norway, ${ }^{5}$ Research School of Finance, Actuarial Science and Statistics, Australian National University, Canberra, Australia, ${ }^{6}$ Department of Statistics and Data Science, Department of Computational Biology, Cornell, Ithaca, USA, ${ }^{7}$ Department of Mathematics and Statistics, University of Limerick, Limerick, Ireland.

Please cite as: Warmenhoven J, Harrison AJ, Quintana DS, Hooker G, Gunning E, Bargary N. Unlocking sports medicine research data while maintaining participant privacy via synthetic datasets. (2020) SportRXiv. https://doi.org/XX.XXXX/osf.io/XXXXX

\section{Research within crisis}

The world is experiencing unprecedented health, economic, and environmental shock due to the COVID-19 global pandemic. COVID-19 poses an enormous burden on scientists continuing necessary components of research practice. Early career researchers (ECR) and PhD students are disproportionately affected ${ }^{1}$, many of these scholars reporting that COVID-19 lockdowns are hindering their ability to collect data, discuss research and disseminate findings. With only $12 \%$ of final-year doctoral students reporting institutional support for extension of

All authors have read and approved this version of the manuscript. This article was last modified on August 17, 2020.
Authors JS @johnwarmenhoven, DQ @dsquintana, EG @gunning_edward, GH @gileshooker \& NB @NormaBargary, can be reached on Twitter. 
studies ${ }^{1}$, this leaves fewer opportunities to meet the data collection requirements of research programs.

\section{The power of synthetic data}

One potential solution is to leverage open datasets for generation of new knowledge and hypotheses. Despite growing availability of open access datasets in sports medicine, ethical and privacy issues can limit the practice of releasing data to the research community. This is problematic for previously collected datasets that were obtained ethically without the intention of making them open access. The creation of synthetic datasets, which "mimic" an original dataset while maintaining privacy of participants, could provide a solution for unlocking otherwise inaccessible data. Synthetic data are partially generated from models that preserve important statistical properties and relationships between variables, allowing researchers to fit exploratory data analysis (EDA) models to the synthetic data, which can be verified by the custodians of the original data. Quintana ${ }^{2}$ demonstrated this concept using three biobehavioural science datasets and the Synthpop R package ${ }^{3}$, to create their synthetic replicates. Analyses of these synthetic datasets yielded almost identical results to the original datasets, noted through a high degree of overlap in regression coefficient confidence intervals generated by the original and synthetic datasets. This high overlap was present regardless of the size of the original dataset, the number of missing data points and the skewness of the data. Synthpop provides several methods for mimicking real datasets, but there is also an opportunity to collaborate with the statistics community for developing other alternative ways of creating synthetic data that are aligned with different data-contexts (i.e. data properties, model frameworks, dimensionality, etc.).

Research conducted on synthetic data could be exploratory leading to construction of new hypotheses. This process could draw on a range of EDA techniques inclusive of data visualization, iterative model specification and model re-specification to find patterns and reveal data structure. ${ }^{4}$ This can also draw on non-inferential methods like exploratory factor analysis and principal components analysis, for extracting multivariate patterns. Additionally, exploratory analyses can be verified using the original datasets by the data custodians, who could co-author manuscripts reporting the results of exploratory analysis.

\section{A use case for sports medicine}

An example of application for sports medicine is the exploration of biomechanics relative 
to injury. Currently, conjecture surrounds the usefulness of biomechanical screening for prediction of injuries. This has included critique of athlete screening tests for injury prediction, with $\mathrm{Bahr}^{5}$ stating that more appropriate statistical measures should be explored to describe the predictive ability of screening tests, including likelihood ratios or receiver operating characteristic curve analyses. Biomechanics has, however extended beyond the use of such discrete approaches, towards continuum-based frameworks such as Functional Data Analysis $(\text { FDA })^{6}$ and Statistical Parametric Mapping (SPM). ${ }^{7}$ For both FDA and SPM, whole curves represent the entirety of movement during a task and treated as a single entity for statistical analysis. Both frameworks provide avenues for exploring patterns, forming predictions and testing directional hypotheses with continuum-based data. There are also examples, where original datasets have been revisited using FDA, with new findings emerging relative to retrospective $A C L$ rehabilitation data. ${ }^{8}$ Designing ways to create and leverage synthetic datasets in this context would open up possibilities on a broader scale for biomechanics and injury, making experimental datasets available to the research community for exploration using these statistical approaches.

\section{Moving forwards...}

There is an opportunity for sports medicine to progress research ideation using synthetic datasets, permitting ECRs to progress research investigations and maintain research momentum when data collection is not feasible. These processes maximise the potential for application of open science practice, while retaining the commitment to privacy and ethics. Research data takes time, effort and money to collect, thus efficient use should be made of data collection in anticipation of the economic fallout of events like COVID-19. Synthetic data could lead to a form of sustainable data analysis for future research, focusing on two areas:

1. Care should be taken developing and implementing methods for constructing synthetic data, by engaging the statistics and/or data science communities to ensure synthetic data are accurate. Clear documentation should also be provided on the model frameworks used to generate the data.

2. EDA can be applied to vetted synthetic datasets to frame new hypotheses that can be tested as a part of future confirmatory research.

Regardless, the potential of synthetic data should be explored further for sports medicine. 


\section{Contributions}

Given this was a conceptual piece of work and did not use data at any stage in the piece of work, no contributions are provided fort acquisition of data or analysis and interpretation of data.

Contributed to conception and design: JW, DH, DQ, EG, GH \& NB.

Drafted and/or revised the article: JW, DH, DQ, EG, GH \& NB.

Approved the submitted version for publication: JW, DH, DQ, EG, GH \& NB.

\section{Acknowledgements}

No additional acknowledgements.

\section{Funding information}

Dr. Daniel S. Quintana's research is supported by an Excellence Grant from the Novo Nordisk Foundation (NNF16OC0019856). Dr. Norma Bargary is supported in part by Grants from Science Foundation Ireland (Grant No. 12/RC/2289-P2, 16/RC/3918, 12/RC/2275_P2, 18/CRT/6049) and co-funded under the European Regional Development Fund.

\section{Data and Supplementary Material Accessibility}

No data was used as a part of the construction of this article and no supplementary information was constructed in conjunction with this article.

\section{REFERENCES}

1. Byrom N. COVID-19 and the Research Community: The challenges of lockdown for earlycareer researchers. Elife. 2020; 9:e59634.

2. Quintana DS. A synthetic dataset primer for the biobehavioural sciences to promote 
reproducibility and hypothesis generation. Elife. 2020; 9:e53275.

3. Nowok B, Raab GM, Dibben C. synthpop: Bespoke creation of synthetic data in R. Journal of Statistical Software. 2016; 74(11):1-26.

4. Behrens JT. Principles and procedures of exploratory data analysis. Psychological Methods. 1997; 2(2):131.

5. Bahr R. Why screening tests to predict injury do not work-and probably never will...: a critical review. British Journal of Sports Medicine. 2016:bjsports-2016-096256.

6. Ramsay J, Silverman B. Functional Data Analysis, 2nd ed, New York, Springer; 2005.

7. Pataky TC, Robinson MA, Vanrenterghem J. Vector field statistical analysis of kinematic and force trajectories. Journal of Biomechanics. 2013; 46(14):2394-2401.

8. Hébert-Losier K, Pini A, Vantini $S$, et al. One-leg hop kinematics 20 years following anterior cruciate ligament rupture: Data revisited using functional data analysis. Clinical Biomechanics. 2015; 30(10):1153-1161. 